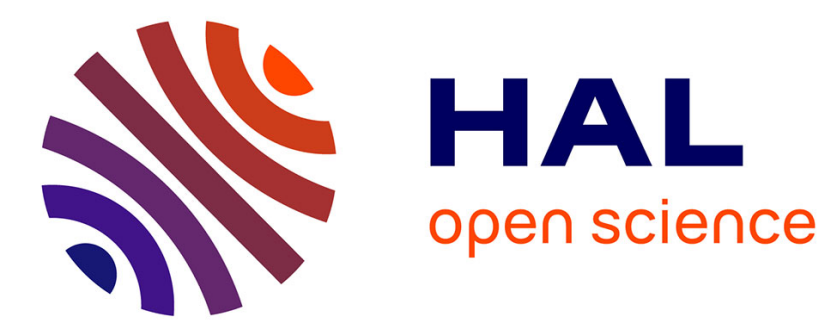

\title{
Multifractal Clustering in Compressible Flows
}

Jérémie Bec, Krzysztof Gaw dzki, Péter Horvai

\section{To cite this version:}

Jérémie Bec, Krzysztof Gaw dzki, Péter Horvai. Multifractal Clustering in Compressible Flows. Physical Review Letters, 2003, 92 (22), 10.1103/PhysRevLett.92.224501 . hal-01399318

\section{HAL Id: hal-01399318 https://hal.science/hal-01399318}

Submitted on 12 Jan 2022

HAL is a multi-disciplinary open access archive for the deposit and dissemination of scientific research documents, whether they are published or not. The documents may come from teaching and research institutions in France or abroad, or from public or private research centers
L'archive ouverte pluridisciplinaire HAL, est destinée au dépôt et à la diffusion de documents scientifiques de niveau recherche, publiés ou non, émanant des établissements d'enseignement et de recherche français ou étrangers, des laboratoires publics ou privés. 


\title{
Multifractal Clustering in Compressible Flows
}

\author{
Jérémie Bec, ${ }^{1,2,3}$ Krzysztof Gawȩdzki, ${ }^{4,1}$ and Péter Horvai ${ }^{5,1,6}$ \\ ${ }^{1}$ Institute for Advanced Study, Einstein Drive, Princeton, New Jersey 08540, USA \\ ${ }^{2}$ Département Cassiopée, Observatoire de la Côte d'Azur, BP 4229, 06304 Nice Cedex 4, France \\ ${ }^{3}$ Dipartimento di Fisica, Università La Sapienza, P. le A. Moro 2, 00185 Roma, Italy \\ ${ }^{4}$ CNRS, Laboratoire de Physique, ENS-Lyon, 46 Allée d'Italie, 69364 Lyon Cedex 7, France \\ ${ }^{5}$ Centre de Physique Théorique, École Polytechnique, 91128 Palaiseau Cedex, France \\ ${ }^{6}$ Laboratoire de Physique, ENS-Lyon, 46 Allée d'Italie, 69364 Lyon Cedex 7, France
}

(Received 13 October 2003; published 4 June 2004)

\begin{abstract}
A quantitative relationship is found between the multifractal properties of the asymptotic mass distribution in a random dissipative system and the long-time fluctuations of the local stretching rates of the dynamics. It captures analytically the fine aspects of the strongly intermittent clustering of dynamical trajectories. Applied to a simple compressible hydrodynamical model with known stretching-rate statistics, the relation produces a nontrivial spectrum of multifractal dimensions that is confirmed numerically.
\end{abstract}

DOI: 10.1103/PhysRevLett.92.224501

During the last ten years important progress has been achieved on the issue of anomalous scaling in passive advection by turbulent velocities. Tools borrowed from high-energy and statistical physics have been used to explain how scaling not predicted by dimensional analysis arises in the dynamics with pumping of tracer particles carried by prescribed incompressible turbulent flow, such as in the Kraichnan model [1] (see, e.g., the review [2]).

Much more violent phenomena, not requiring any pumping, take place in compressible flows or, more generally, for dissipative particle dynamics. The trajectories may then concentrate on dynamically evolving singular sets with fractal and multifractal properties. An example with applications to rain-droplet growth [3], chemical reactions in chaotic flows [4], and plankton dynamics [5] is provided by suspensions of inertial particles of finite size subject to Stokes drag and therefore described by a dissipative dynamics even in an incompressible flow [6]. The strong intermittency of the density fluctuations characterized by multifractal concentrations has to be taken into account in estimates of spore contamination risks or of the speed of chemical processes. While trying to relate the multifractal properties of inertial particles to the fluctuations of Lyapunov exponents of the associated particle trajectories, we came across a novel relationship which is applicable to many dissipative systems. After formulating the general problem, we give a semiheuristic derivation of this relation. We then consider a compressible variant of the Kraichnan model for which the multifractal properties can be determined by alternative means (both theoretically and numerically) in order to validate the proposed relation. Detailed applications to inertial particles will be discussed elsewhere.

As a general model of dissipative dynamics, consider the equation
PACS numbers: $47.53 .+\mathrm{n}, 05.40 .-\mathrm{a}, 05.45 .-\mathrm{a}, 47.52 .+\mathrm{j}$

$$
\frac{d}{d t} \boldsymbol{X}(t, \boldsymbol{x})=\boldsymbol{v}(t, \boldsymbol{X}(t, \boldsymbol{x})), \quad \boldsymbol{X}(0, \boldsymbol{x})=\boldsymbol{x},
$$

for particle trajectories in a bounded region, where $\boldsymbol{v}$ is a compressible velocity field. We take $\boldsymbol{v}$ random with a prescribed statistics. The transport of a density field $\rho$ in such dynamics is governed by the continuity equation

$$
\partial_{t} \rho+\nabla \cdot(\rho \boldsymbol{v})=0 .
$$

If the initial density at time $t_{0}$ is uniform and if the flow is compressible and sufficiently mixing, then the solution $\rho(t)$ of (2) will approach, when $t_{0}$ tends to $-\infty$, a singular limit $\rho_{*}(t)$ which is a measure with support on the dynamical attractor towards which the Lagrangian trajectories converge. For random velocities, the measure $\rho_{*}(t)$ and the dynamical attractor depend on the velocity realization. We consider stationary velocity ensembles where the statistics of $\rho_{*}(t)$ does not depend on $t$. For convenience we consider $t=0$ and denote $\rho_{*}(0)=\rho_{*}$. One expects the measure $\rho_{*}$ to have roughly a local product structure with a smooth density along the unstable manifolds of the flow and a fractal-like structure in the transverse directions (these properties are those of the Sinai-Ruelle-Bowen measures [7]).

The Lagrangian average defined by

$$
\langle F\rangle \equiv \overline{\int F(\boldsymbol{x} \mid \boldsymbol{v}) \rho_{*}(\boldsymbol{x} \mid \boldsymbol{v}) d \boldsymbol{x}},
$$

where the overline denotes averaging with respect to the velocity ensemble, samples points on the random attractor according to the density $\rho_{*}$ of the asymptotic tracer distribution.

We are interested in the small-scale statistics of the mass distribution associated to the measure $\rho_{*}$. Denote by $\mathcal{B}_{r}(\boldsymbol{x})$ the ball of radius $r$ around the point $\boldsymbol{x}$ and consider the mass $m_{r}(\boldsymbol{x})=\int_{\mathcal{B}_{r}(\boldsymbol{x})} \rho_{*}$ contained in it. We expect the 
Lagrangian-averaged powers of $m_{r}$ to exhibit the small- $r$ scaling

$$
\left\langle m_{r}^{n}\right\rangle \sim r^{\xi_{n}} .
$$

The function $\operatorname{HP}(n)=\xi_{n-1} /(n-1)$ is usually called the Hentschel-Procaccia spectrum for dimensions [8]. A nonlinear dependence on $n$ of the scaling exponents $\xi_{n}$ implies intermittency in the mass distribution, in particular, the presence of non-Gaussian tails in the probability density function (PDF) of $m_{r}$. The scaling behavior (3) suggests that the PDFs of $h_{r} \equiv \ln m_{r} / \ln r$ take, for small radii, the large deviations form $e^{(\ln r) S(h)}$ with the rate function $S(h)$ and the scaling exponents $\xi_{n}$ related by the Legendre transform: $S(h)=\max _{n}\left[\xi_{n}-n h\right]$. Below, we obtain a precise expression for the scaling exponents $\xi_{n}$ in terms of the large deviations of the stretching rates of the flow. We note here that in [9] a similar relation was discussed for $n=1$ and, in a qualitative way, for large $n$.

In order to define the stretching rates, consider the matrix $W(t, \boldsymbol{x}) \equiv\left(\partial_{j} X^{i}(t, \boldsymbol{x})\right)$ describing the flow linearized around a given trajectory $\boldsymbol{X}(t, \boldsymbol{x})$ satisfying (1). The eigenvalues of the positive matrix $W^{T} W$ may be written, arranged in nonincreasing order, as $e^{2 t \sigma_{1}}, \ldots, e^{2 t \sigma_{d}}$ with $\sigma_{i} \equiv \sigma_{i}(t, \boldsymbol{x} \mid \boldsymbol{v})$ called the stretching rates of the flow. The limits $\lambda_{i} \equiv \lim _{t \rightarrow \infty} \sigma_{i}(t)$ exist almost surely with respect to the Lagrangian sampling and are constant if the system is ergodic. They define the Lyapunov exponents of the flow. This is the content of the multiplicative ergodic theorem [10]. As argued in [11], when the exponents $\lambda_{i}$ are all different, the PDF of the stretching rates takes, for large $t$, the large deviations form ( $\theta$ denotes the Heaviside function)

$$
e^{-t H\left(\sigma_{1}, \ldots, \sigma_{d}\right)} \theta\left(\sigma_{1}-\sigma_{2}\right) \cdots \theta\left(\sigma_{d-1}-\sigma_{d}\right)
$$

with convex rate function $H$ attaining its minimum, equal to zero, at $\sigma_{i}=\lambda_{i}$.

When the top Lyapunov exponent $\lambda_{1}$ is negative, the attractor measure $\rho_{*}$ degenerates into points with trivial mass statistics. For $\lambda_{1}>0$, it was shown in [12] that the local dimension $h=\lim _{r \rightarrow 0} h_{r}$ is almost surely equal to the Lyapunov dimension $d_{L}=j+\delta$, where $0<\delta \leq 1$ is such that $\lambda_{1}+\cdots+\lambda_{j}+\delta \lambda_{j+1}=0$. The above statement implies that

$$
d \xi_{n} /\left.d n\right|_{n=0}=d_{L} .
$$

The Lyapunov dimension, introduced by Kaplan and Yorke [13], may be heuristically interpreted as the dimension of objects keeping a constant volume during time evolution. Of course, Eq. (5) gives only partial information about the mass scaling as compared to the full set of exponents $\xi_{n}$.

To relate the mass distribution in small balls to the fluctuations of the stretching rates, we focus on the twodimensional case. The expected smoothness of $\rho_{*}$ in the unstable direction leads us to consider small parallelograms with one side of size $r_{1}$ parallel to the unstable manifold and with extension $r_{2}$ in the direction perpen- dicular to it. We expect that the moments of the mass $m_{r_{1}, r_{2}}$ in such parallelograms obey

$$
\left\langle m_{r_{1}, r_{2}}^{n}\right\rangle \sim r_{1}^{n} r_{2}^{\xi_{n}-n}
$$

for small $r_{1}, r_{2}$ as long as $\xi_{n}-n \geq 0$. When $\xi_{n}<n$, we expect (6) to be replaced for $r_{2}>r_{1}$ by

$$
\left\langle m_{r_{1}, r_{2}}^{n}\right\rangle \sim r_{1}^{\xi_{n}} r_{2}^{0} .
$$

This may be viewed as analogous to the stretching in one direction of a fractal set of dimension $D$, while contracting it in the other one. In the expanding direction, the set behaves (down to a scale depending on the stretching) as if projected on a line, so that its dimension in this direction is $\min (1, D)$. In the other direction it has the complementary dimension $D-\min (1, D)=$ $\max (D-1,0)$.

We consider such a parallelogram at $t>0$ and look at its preimage at time zero. While the direction parallel to the unstable manifold is exponentially contracted backward in time with a rate given by the largest stretching rate $\sigma_{1}$, the other direction typically expands with an exponential rate $\sigma_{2}$. Hence the time-zero preimage of the original parallelogram is (approximately) another parallelogram as sketched in Fig. 1 .

Conservation of mass and stationarity of the statistics lead to the relation

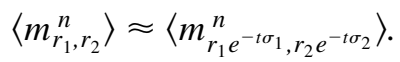

If there is a sufficiently rapid loss of memory in the Lagrangian evolution (i.e., if the Lagrangian dynamics is sufficiently mixing) then the expectation on the righthand side should factorize for large $t$ (such a factorization holds for all $t$ in the Kraichnan model discussed below). In such a situation, using the large deviation form (4) of the PDF of the stretching rates, we infer that

$$
\left\langle m_{r_{1}, r_{2}}^{n}\right\rangle \sim \int_{\sigma_{1} \geq \sigma_{2}}\left\langle m_{r_{1}}^{n} e^{-t \sigma_{1}, r_{2} e^{-t \sigma_{2}}}\right\rangle e^{-t H\left(\sigma_{1}, \ldots, \sigma_{2}\right)} d \sigma_{1} d \sigma_{2} .
$$

Consistency of the above relation with the scaling (6) requires that

$$
1 \sim \int_{\sigma_{1} \geq \sigma_{2}} e^{-t\left[n \sigma_{1}+\left(\xi_{n}-n\right) \sigma_{2}+H\left(\sigma_{1}, \sigma_{2}\right)\right]} d \sigma_{1} d \sigma_{2} .
$$

Since $t$ is assumed large, a saddle-point argument implies then the following relation between the scaling exponents

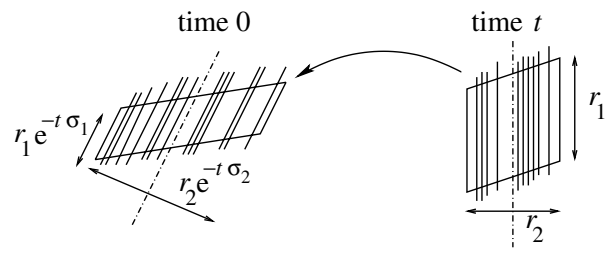

FIG. 1. Sketch of the backward-in-time evolution between times $t$ and 0 of a small parallelogram of length $r_{1}$ in the direction of the unstable manifold and $r_{2}$ in the perpendicular direction. 
$\xi_{n}$ and the rate function $H$ of the stretching rates:

$$
\min _{\sigma_{1} \geq \sigma_{2}}\left[n \sigma_{1}+\left(\xi_{n}-n\right) \sigma_{2}+H\left(\sigma_{1}, \sigma_{2}\right)\right]=0 .
$$

Alternative formulations are

$$
\xi_{n}=n-\max _{\sigma_{1} \geq \sigma_{2}} \frac{1}{\sigma_{2}}\left[n \sigma_{1}+H\left(\sigma_{1}, \sigma_{2}\right)\right]=\min _{h \geq 0}[h n+S(h)]
$$

in which $S(h)=\min _{\sigma>0} \sigma^{-1} H((h-1) \sigma,-\sigma)$. These formulas are valid for $\xi_{n} \geq n$. Similarly, for $\xi_{n} \leq n$, we obtain from (7)

$$
\xi_{n}=-\max _{0>\sigma_{1} \geq \sigma_{2}} \frac{1}{\sigma_{1}} H\left(\sigma_{1}, \sigma_{2}\right) .
$$

The above relations between the exponents $\xi_{n}$ and the rate function $H$ constitute the main result of this Letter.

It is easily checked from (8) that $\left.\left(d \xi_{n} / d n\right)\right|_{n=0}=1-$ $\lambda_{1} / \lambda_{2}$, thereby recovering (5). Also from (8) one can show that $\lim _{t \rightarrow \infty} t^{-1}\left\langle\left|W(t, \boldsymbol{x}) \boldsymbol{R}_{0}\right|^{-\xi_{1}}\right\rangle=0$, for any initial separation $\boldsymbol{R}_{0}$, meaning that the generalized Lyapunov exponent of order $-\xi_{1}$ vanishes. This is a known result for smooth stochastic flows [14]. Relations (8) and (9) can be extended with the use of similar arguments to dimensions higher than two by investigating the scaling properties of the mass distribution in small parallelepipeds aligned with subspaces of less and less unstable directions of the dynamics.

To illustrate and further validate the relation, we now consider a special class of compressible velocity fields chosen as a variant of the Kraichnan model [1]. For the domain we take a periodic box. The velocity $\boldsymbol{v}$ is centered Gaussian, with covariance

$$
\overline{\boldsymbol{v}^{i}(\boldsymbol{x}+\boldsymbol{\ell}, t+\tau) \boldsymbol{v}^{j}(\boldsymbol{x}, t)}=2\left[D_{0} \delta^{i j}-d^{i j}(\ell)\right] \delta(\tau),
$$

where, for small separations $\ell$, the function $d^{i j}$ satisfies

$$
d^{i j}(\ell)=\frac{D_{1}}{2}\left[(d+1-2 \wp) \delta^{i j} \ell^{2}+2(\wp d-1) \ell^{i} \ell^{j}+o\left(\ell^{2}\right)\right]
$$

assuring local isotropy. The parameter $\wp$, called the compressibility degree, is chosen in the interval $[0,1]$. Its extreme values correspond, respectively, to incompressible and to potential velocity fields. For such velocity ensembles, the distribution of the stretching rates is explicitly known [2]. The corresponding rate function takes the simple form

$$
H=\frac{1}{C_{1}}\left[\sum_{i=1}^{d}\left(\sigma_{i}-\lambda_{i}\right)^{2}+C_{2}\left(\sum_{i=1}^{d}\left(\sigma_{i}-\lambda_{i}\right)\right)^{2}\right] .
$$

$C_{1} \equiv 4 D_{1}(d+\wp(d-2)), C_{2} \equiv(1-\wp d) /[\wp(d-1)(d+$ $2)]$. The Lyapunov exponents are $\lambda_{j}=D_{1}[d(d-2 j+$ 1) $-2 \wp(d+(d-2) j)]$. In the two-dimensional case, the largest Lyapunov exponent is negative when $\wp>$ $1 / 2$. For $\wp<1 / 2$, the exponents for the mass distribution obtained from (8) and (9) read

$$
\xi_{n}= \begin{cases}\frac{2 n+\sqrt{(1+2 \wp)^{2}-8 \xi n^{2}}}{1+2 \xi}-1 & \text { if } n \leq n_{\mathrm{cr}}, \\ \xi_{\infty} & \text { if } n \geq n_{\mathrm{cr}}\end{cases}
$$
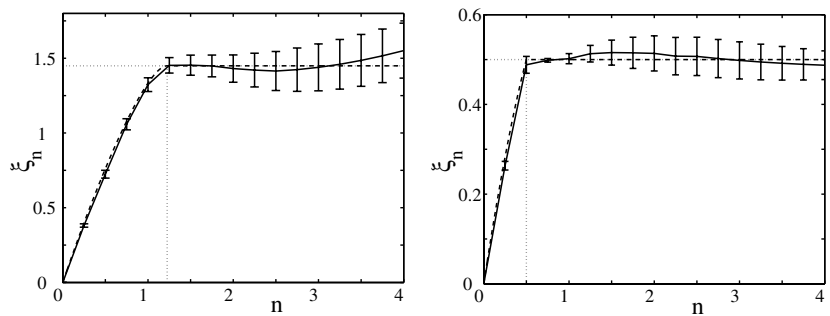

FIG. 2. Scaling exponents $\xi_{n}$ for the mass distribution associated to the advection of a density field by a Kraichnan velocity field. For two different degrees of compressibility, the exponents obtained match those predicted by theory which are represented as dashed lines.

where the critical moment $n_{\mathrm{cr}}$ and the saturation exponent $\xi_{\infty}$ are given by

$$
\begin{aligned}
& \left\{\begin{array}{l}
n_{\mathrm{cr}}=\frac{1}{2} \sqrt{1+\frac{1}{2 \xi}} \\
\xi_{\infty}=2 n_{\mathrm{cr}}-1
\end{array} \quad \text { if } 0<\wp \leq 1 / 6,\right. \\
& n_{\mathrm{cr}}=\xi_{\infty}=\xi_{1}=\frac{2-4 \wp}{1+2 \wp} \quad \text { if } 1 / 6 \leq \wp<\frac{1}{2} \text {. }
\end{aligned}
$$

Note that the saturation of the exponents which governs the high-order statistics is due to extremely rare events for which a mass of order unity is concentrated inside the small ball.

Numerical simulations confirm the scaling exponents obtained above for the compressible Kraichnan model. To distinguish the two cases, two different values of the compressibility degree are investigated $(\wp=1 / 10<$ $1 / 6$ and $\wp=3 / 10>1 / 6$ ). The velocity field $\boldsymbol{v}$ is generated by the superposition of nine independent Gaussian modes and the density is approximated by considering a large number of Lagrangian tracers. The exponents obtained numerically after averaging over $10^{5}$ turnover times and for $N=10^{5}$ tracers are shown in Fig. 2. Although statistical convergence of the average is quite slow, these expensive simulations are in a rather good agreement with the theory, in particular, with the saturation of the exponents after the critical order.

Determination of the exponents is improved for positive integer orders $n$ by considering only $n+1$ particles. This method allows one to perform very long-time averages (here of the order of $10^{8}$ turnover times) required for good convergence of the statistics at small scales. As shown in Fig. 3, for $\wp=3 / 10$, the moments clearly scale over several decades. For the lower value of compressibility $\wp=1 / 10$, convergence is slower because of the smaller probability for the tracked particles to come close together.

Finally, let us mention that, for integer orders, the exponent $\xi_{n}$ can be linked to the homogeneity degree of the stationary single-time density correlation function

$$
\mathcal{F}_{n+1} \equiv \overline{\rho(\mathbf{0}) \rho\left(\boldsymbol{x}_{1}\right) \cdots \rho\left(\boldsymbol{x}_{n}\right)} .
$$

The $n$th order moment of $m_{r}$ may be written as 


$$
\left\langle m_{r}^{n}\right\rangle=\int_{\mathcal{B}_{r}(\mathbf{0}) \times \cdots \times \mathcal{B}_{r}(\mathbf{0})} \mathcal{F}_{n+1}\left(\mathbf{0}, \boldsymbol{x}_{1}, \ldots, \boldsymbol{x}_{n}\right) d \boldsymbol{x}_{1} \cdots d \boldsymbol{x}_{n}
$$

In the particular case of the Kraichnan model, $\mathcal{F}_{n+1}$ is a zero mode of the operator (see, e.g., [2])

$$
M_{n+1}^{\dagger} \equiv \sum_{0 \leq k, \ell \leq n} \partial_{x_{k}^{i}} \partial_{x_{\ell}^{j}}\left[\left(D_{0} \delta^{i j}-d^{i j}\left(\boldsymbol{x}_{k}-\boldsymbol{x}_{\ell}\right)\right)\right] .
$$

We need not write these zero modes explicitly. The homogeneity degree of the isotropic solution of lowest degree can be found simply by requiring its positivity and imposing on it certain continuity and integrability conditions. The branch $n<n_{\mathrm{cr}}$ in (10) is obtained by requiring the solution to be continuous at $\boldsymbol{x}_{1}=\cdots=\boldsymbol{x}_{n}$. On this subspace, $M_{n+1}^{\dagger}$ is degenerate, owing to the fact that collinear points remain collinear when transported by the (linearized) flow. If in addition we restrict $M_{n+1}^{\dagger}$ to the rotationally invariant sector, it becomes an ordinary second-order homogeneous differential operator. It has two scaling solutions. The one with exponent $\xi_{n}-n d$ gives, through (11), the nonsaturated $\left(n<n_{\text {cr }}\right)$ branch of (10). Such a solution breaks down when the corresponding zero mode ceases to be integrable at small $\boldsymbol{x}_{i}$ which may occur if the restriction of the zero mode to the collinear sector is not integrable near 0 , i.e., $\xi_{n}-n d \leq-1$. This gives $n_{\text {cr }}$ and $\xi_{\infty}$ in the case $\wp<1 / 6$. Another scenario is if the zero mode has a nonintegrable singularity around the collinear geometry. This gives $n_{\text {cr }}$ and $\xi_{\infty}$ in the case $\wp>1 / 6$.

In short, even a very simple statistics of the large deviations of stretching rates (a Gaussian one) can give rise to a quite nontrivial multifractal spectrum of the asymptotic density distribution with saturated exponents $\xi_{n}$ signaling violent fluctuations of the concentration.

An important open question not touched upon by this Letter concerns the case of rough-in-space velocity fields appearing in the limit of very high Reynolds numbers. That problem cannot be formulated in terms of stretching
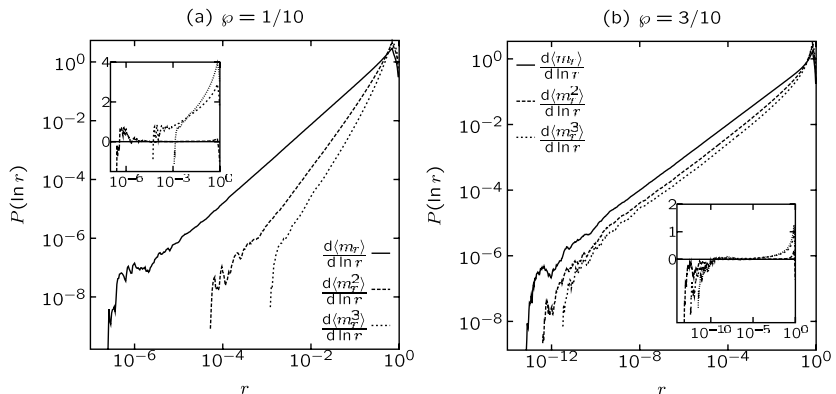

FIG. 3. For $n+1$ particles $(n=1,2$, and 3$)$ the distribution of the maximum of the distances of the reference particle to the other $n$ particles is represented. It can be shown that this PDF is proportional to $d\left\langle m_{r}^{n}\right\rangle / d \ln r$. The insets show the difference with small- $r$ asymptotes with slopes given by the theoretical exponents. rates, but the relationship with density correlations and zero modes still holds.

Let us conclude with some possible applications of the relations between the multifractal properties and the large deviations of the stretching rates. As we have seen, measuring numerically the scaling exponents by particle tracking methods requires very long-time averages. This is due to the extreme rarity of the events dominating large order moments at small spatial scales. The stretching rates capture directly the dynamics at infinitesimal scales and estimating their large deviations together with relations (8) and (9) will generally be a much more efficient method.

We are grateful to D. Dolgopyat, U. Frisch, K. Khanin, Y. Le Jan, and O. Raimond for interesting and motivating discussions. During this work J. B. was supported by the National Science Foundation under Agreement No. DMS-9729992 and by the Indo-French Centre for the Promotion of Advanced Research (IFCPAR 2404-2). K. G. acknowledges support from the von Neumann Fund at IAS in Princeton. This work has been partly done within the ECTMR network under Contract No. HPRNCT-2002-00300. Part of the numerical simulations were performed in the framework of the SIVAM project at the Observatoire de la Côte d'Azur. We also wish to acknowledge that, parallelly but independently from us, A. Fouxon has also obtained, in an as yet unpublished work, relationship (8) between the scaling exponents of mass and the large deviations of the stretching rates.

[1] R. H. Kraichnan, Phys. Rev. Lett. 72, 1016 (1994).

[2] G. Falkovich, K. Gawȩdzki, and M. Vergassola, Rev. Mod. Phys. 73, 913 (2001).

[3] G. Falkovich, A. Fouxon, and M. G. Stepanov, Nature (London) 419, 151 (2002)

[4] A. E. Motter, Y.-C. Lai, and C. Grebogi, Phys. Rev. E 68, 056307 (2003).

[5] T. Nishikawa, Z. Toroczkai, and C. Grebogi, Phys. Rev. Lett. 87, 038301 (2001).

[6] J. Bec, Phys. Fluids 15, L81 (2003).

[7] L.-S. Young, J. Stat. Phys. 108, 733 (2002).

[8] H. G. E. Hentschel and I. Procaccia, Physica (Amsterdam) 8D, 435 (1983).

[9] E. Balkovsky, G. Falkovich, and A. Fouxon, Phys. Rev. Lett. 86, 2790 (2001).

[10] L. Arnold, Random Dynamical Systems (Springer, Berlin, Heidelberg, 1998).

[11] E. Balkovsky and A. Fouxon, Phys. Rev. E 60, 4164 (1999).

[12] F. Ledrappier and L.-S. Young, Commun. Math. Phys. 117, 529 (1988).

[13] J. L. Kaplan and J. A. Yorke, in Lecture Notes in Mathematics (Springer, Berlin, 1979), Vol. 730, p. 204.

[14] P. H. Baxendale, in Spatial Stochastic Processes, Progress in Probability Vol. 19 (Birkhäuser, Boston, 1991), p. 189-218. 